\title{
What we have learned from operational monitoring and serviceability assessment of long-span bridges
}

\author{
Ho-Kyung Kim ${ }^{1}$, Sunjoong Kim², Jin Park ${ }^{3}$, You-Chan Hwang ${ }^{4}$ \\ DOI: https://doi.org/10.5592/CO/BSHM2017.2.3 \\ ${ }^{1,2,4}$ Department of Civil Engineering, Seoul National University (1 Gwanak-ro, Seoul, 08826, Korea, Rep.) \\ ${ }^{3}$ KBRC, Seoul National University (1 Gwanak-ro, Seoul, 08826, Korea, Rep.) \\ E-mails: ${ }^{1}$ hokyungk@snu.ac.kr; ${ }^{2}$ fanta909@snu.ac.kr; ${ }^{3} a z 22 @ s n u . a c . k r ;{ }^{4} l u m 4 u 2526 @ s n u . a c . k r$
}

\begin{abstract}
More than 50 cable-supported bridges have been built in Korea since 1980. While maintaining those infrastructures for last 30 years, we have experienced unexpected problems from operational field monitoring of long-span bridges. This paper discusses on what we have observed, learned and how we responded to those events. As an example, a vortex-induced vibration was observed in 2011 in parallel-disposed twin cable-stayed bridges. Operational monitoring and wind tunnel tests successfully identified that the close distance between two bridges was one of the main cause of the vibration. However, an operational modal analysis revealed that unexpected low damping ratio of the bridge was also contributed to the realization of this serviceability problem in an operating bridge. Another observation of vibration in a long-span suspension bridge also attracted our attention on the potential low damping ratio, which may invoke serviceability issues in assessment of bridges. This paper introduces the details of the cause investigations, assessment and mitigation of the serviceability problems, validation through field tests and operational monitoring, and concluding suggestions to bridge owners and engineers.
\end{abstract}

Keywords: OMA, damping, serviceability, St-Id, structural health monitoring, cable-stayed bridge, suspension bridge, VIV

\section{Introduction}

The Namhae Suspension Bridge, built in 1973, was the starting point of cable-supported bridge history in Korea. Now we are operating 49 cable-supported bridges (43 cable-stayed bridges and 6 suspension bridges) and 23 more bridges (19 cable-stayed bridges and 4 suspension bridges) are in construction as of Sep., 2015. Fig. 1 shows the bridge data in terms of the length of main span and the year of opening to traffic, and Fig. 2 shows the distribution of the bridges over the Korean peninsula.

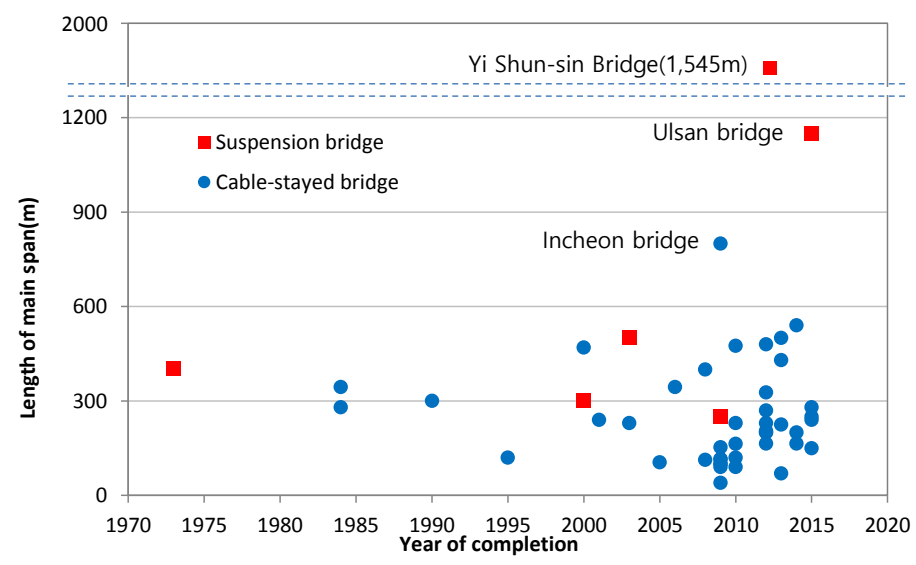

Fig. 1. Data of cable-supported bridges in Korea (Courtesy of TM-ENC)

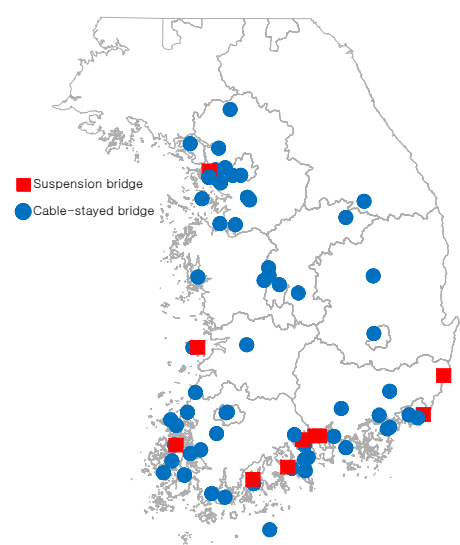

Fig. 2. Locations of cable-supported bridges (Courtesy of TM-ENC)

Most of the long-span bridges adopt built-in sensors for structural health monitoring (SHM) during operation. The data collected from the SHM system are accumulating in database of the bridge owners, as shown 
in Fig. 3. The SHM data of the bridges operated by the Ministry of Land, Infrastructure and Transport, are particularly collected through the Integrated Long-Span Bridge Management Center.
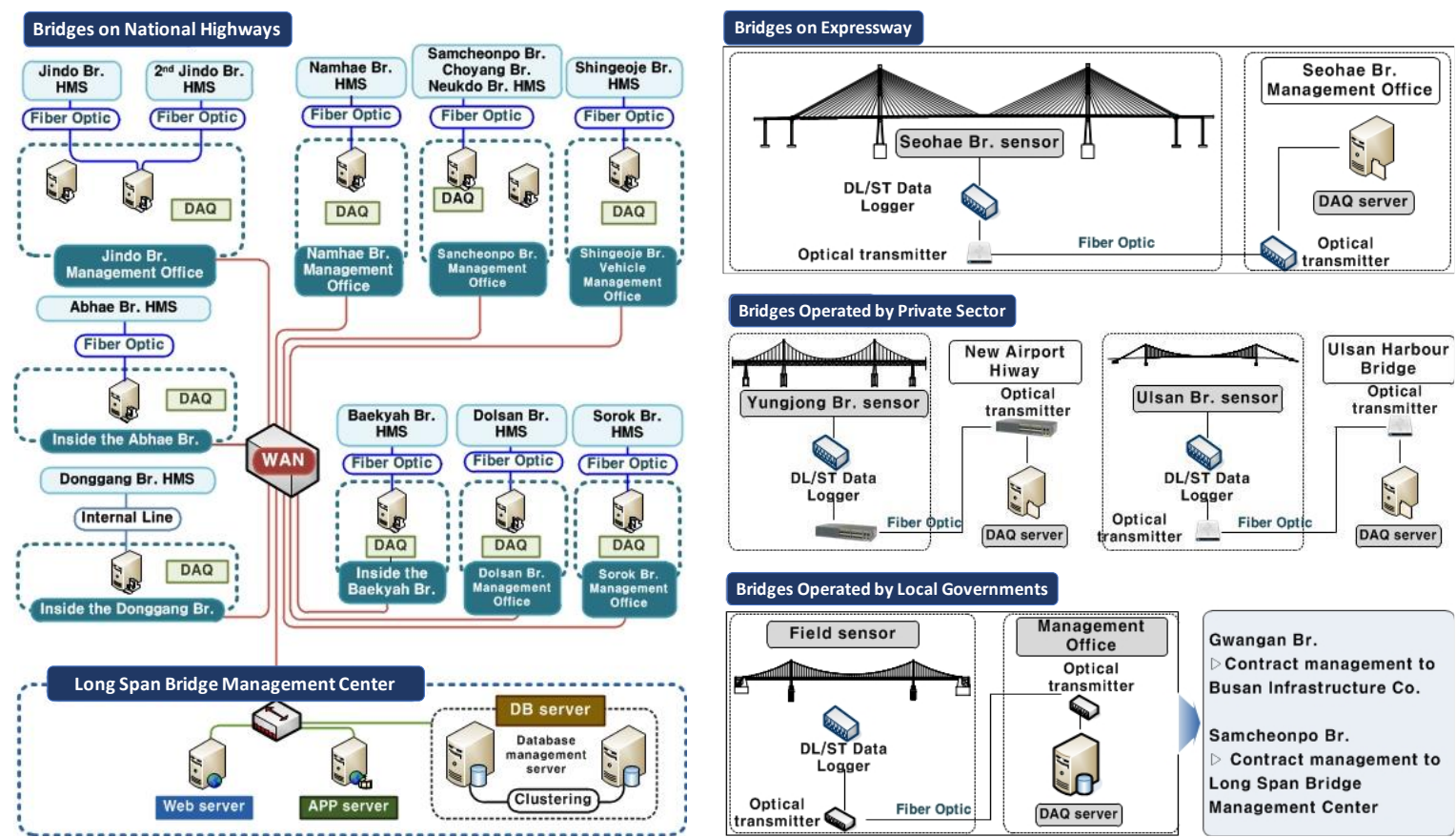

Fig. 3. Data accumulation from SHM system (Courtesy of TM-ENC)

Even though careful consideration has been applied for the best deployment of manageable number of sensors, it does not mean all the time that the best data accumulation and utilization are guaranteed from the viewpoint of more comfortable and safe operation of structures. The SHM has focused on the automatic damage detection techniques for a long time (Brownjohn and Carden, 2007; Magalhães et al., 2012; Peeters and De Roeck, 2001). Even though several successful achievements have been demonstrated, the damage detection also showed intrinsic limitation for large-scale infrastructures. Another value of SHM application should be a structural identification (St-Id) of flexible structures mainly targeting identification of dynamic properties. The natural frequencies and corresponding mode shapes are fundamental properties to be well estimated for the assessment of bridge performance not only in dynamic but even in static point of view. The identification of those two parameters are relatively straightforward from operational modal analysis (OMA) by utilizing data obtained from built-in monitoring sensors and/or densely applied temporal sensors for special purposes. Another dynamic parameter is modal damping ratio that is practically important in assessment of vibrational serviceability performance of flexible structures including long-span bridges. The modal damping ratios can be theoretically estimated by applying sweeping test with exciters for harmonic motion. Specially developed exciters were utilized for excitation of actual bridges (Brownjohn et al., 2003; Chen et al., 2016). A sudden release test of heavy weight was also utilized for the excitation of free-decaying motion of bridge structure (Magalhães et al., 2010). However, as the span length increases in recent long-span and super-long span bridges, the use of heavy exciters or sudden release tests are no more applicable from the point of view not only in structural safety but also in securing continuous traffic flows. Accordingly, OMA is becoming the only choice for St-Id nowadays.

This paper presents two events of vortex-induced vibration (VIV) observed in cable-supported bridges. The cause investigations of those unpredicted events reveal the importance of bridge aerodynamics for huge but also flexible structures. However, a more in-depth consideration of the cause of VIV shed light on St-Id of inherent modal damping ratios for more valuable utilization of SHM for the prognosis of potential vulnerability in serviceability performance of flexible structures. This paper demonstrates the role of damping ratios in observed VIV, the uncertainties in identified damping ratios, suggestions in data processing for more reliable St-Id, and finally concluding suggestions to bridge owners and operating engineers. 

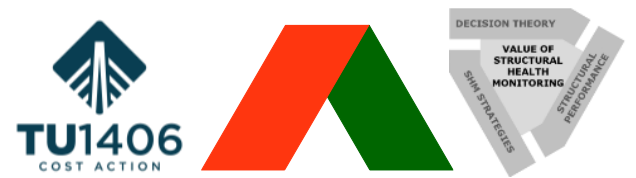

\section{Case Study of VIV in Parallel Cable-Stayed Bridges}

\subsection{Observed VIV}

The Jindo Bridge is composed of two parallel cable-stayed bridges. The first bridge (Bridge 1) was built in 1984 and the second bridge (Bridge 2) was opened to traffic to accommodate the increased volume of traffic in 2005. Two decks are in tandem with a net distance of $9.9 \mathrm{~m}$, as shown in Fig. 4. A noticeable VIV was observed for the deck of Bridge 2, located upstream. The mean wind velocity was maintained as $9.8-11.5 \mathrm{~m} / \mathrm{s}$ for two hours. Since the amplitude of the observed acceleration exceeded the allowable level, as shown in Fig. 5, a series of wind tunnel tests were carried out to identify the main causes of vibration.

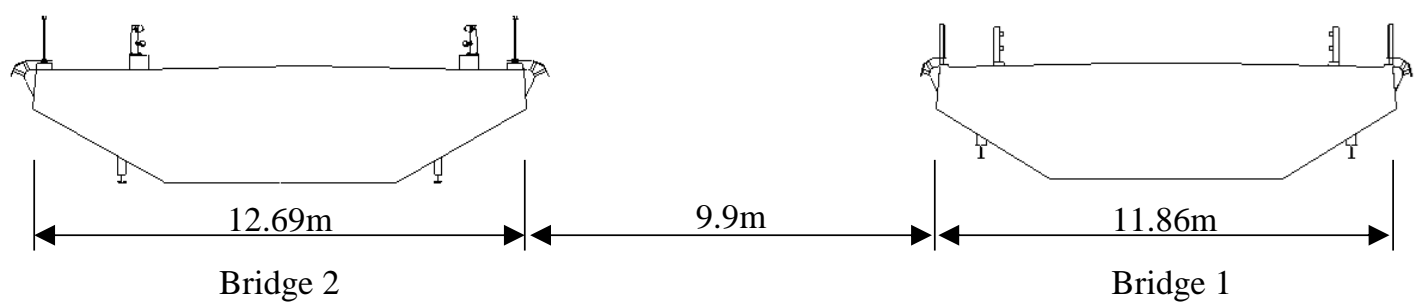

Fig. 4. Two decks of Jindo Bridge in tandem

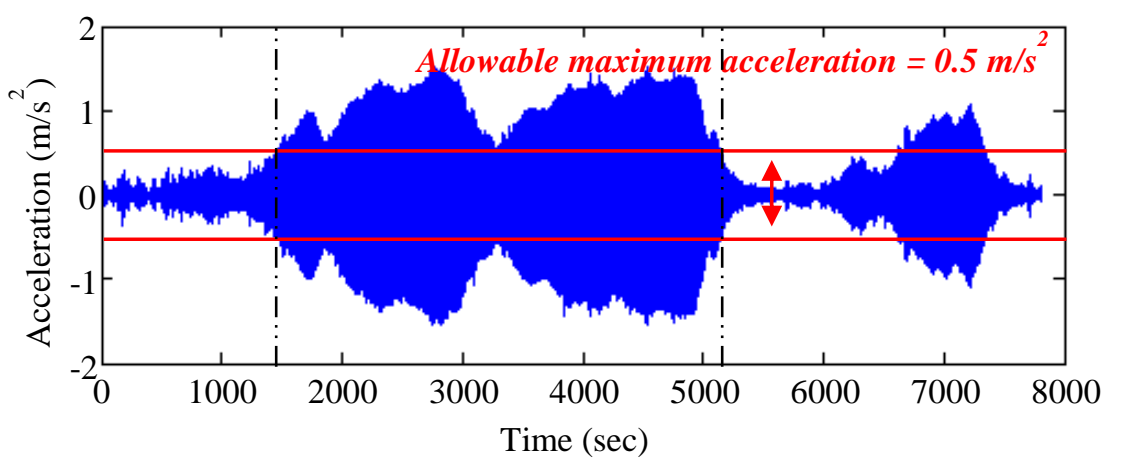

Fig. 5. Vertical acceleration of the deck in Bridge 2 at the center of main span

\subsection{Cause Investigation in a Wind Tunnel}

The purpose of wind tunnel tests was 1) to confirm the VIV in upstream deck being originated from parallel disposition of two decks, 2) to examine any potential aerodynamic measure including modification of external shape in upstream deck, 3) to identify the flow change in between two decks by applying a particle image velocimetry, and eventually 4) to identify the relationship between amplitude of VIV and damping ratio.

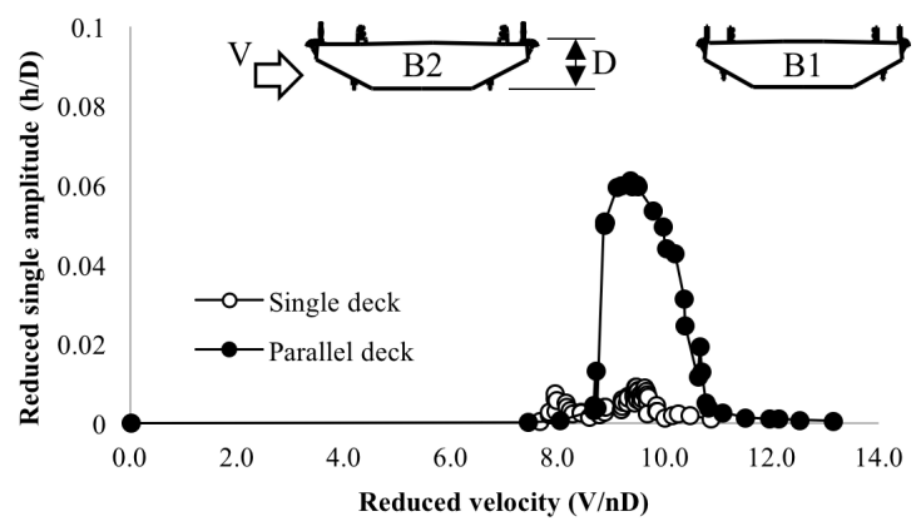

Fig. 6. Amplitude of VIVs in both a single deck and parallel decks 
Fig. 6 compares the amplitudes of lock-in VIV for single deck (only Bridge 2) and parallel decks (Bridge 2 in upstream and Bridge 1 in downstream). It is found that the VIV in the upstream deck was amplified due to the presence of another deck in downstream. Every tries in modification of aerodynamic shape of upstream deck were not effective in mitigating the VIV (Seo et al, 2013).

Fig. 7 shows phase-averaged flow streams between two decks at the lock-in wind velocity of parallel-deck case. The Karman-type vortex trails behind the upstream deck were developed to motion-induced vortices, which resulted in interference VIV in upstream deck (Seo et al, 2013).

(a)

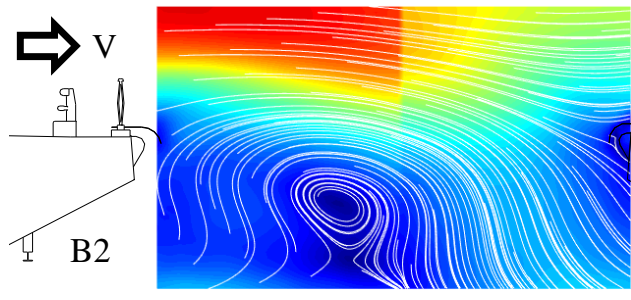

(b)

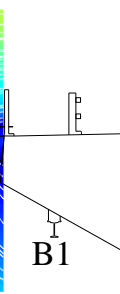

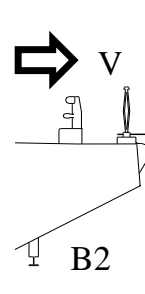

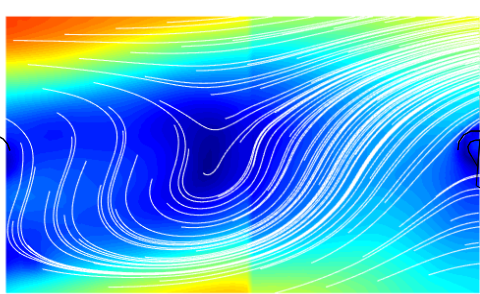

$\mathrm{B}$

Fig. 7. Phase-averaged flow streams at the phase of (a) $\pi / 2$, (b) $3 \pi / 2$

Fig. 8 shows the relationship between the amplitude of VIV in upstream deck to the damping ratio in wind tunnel setup (Seo et al, 2013). Fig. 8 demonstrates the high sensitivity of VIV amplitude to damping ratio. Based on Fig. 8, the field monitored amplitude of VIV of $0.19 \mathrm{~m}$ approximately corresponds to a potential damping ratio of $0.21 \%$, which is much less than the design damping ratio of $0.4 \%$ recommended in the design guidelines (KSCE, 2006).

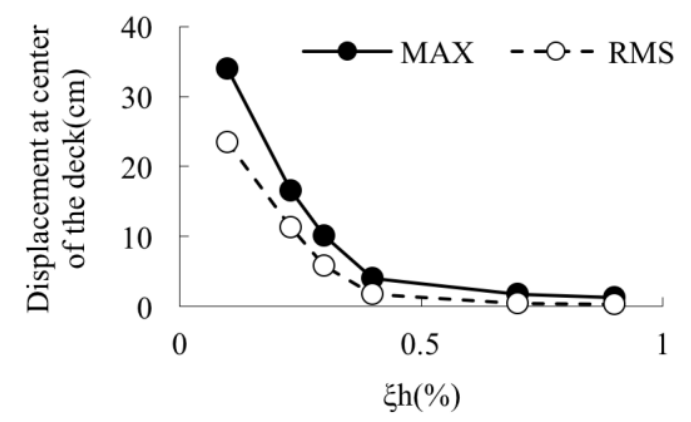

Fig. 8. Amplitude of VIV for set-up damping ratio in wind tunnel

\subsection{OMA-Based Damping Identification}

Based on thorough wind tunnel tests, two main causes were indicated. The first cause was the amplification of VIV in upstream deck owing to the close parallel disposition between two decks, and the second one was unexpected low damping ratio of Bridge 2. The NExT-ERA technique was applied as an OMA to three-day acceleration data measured at the center of mid-span in both bridges (Kim et al., 2013). Fig. 9(a) shows the identified damping ratios for each segmented 20-min data. A large scattering was observable but the mean value of the damping ratio was $0.28 \%$, which showed a somehow agreement with the predicted one in wind tunnel tests. However, the damping ratios for Bridge 1 was identified much higher than those for Bridge 2 by showing around $0.69 \%$, as shown in Fig. 9(b). In fact, the damping ratio is an inherent property of bridge and, even two similar bridges in terms of materials and overall shapes show a quite different levels of damping ratio.

(a)

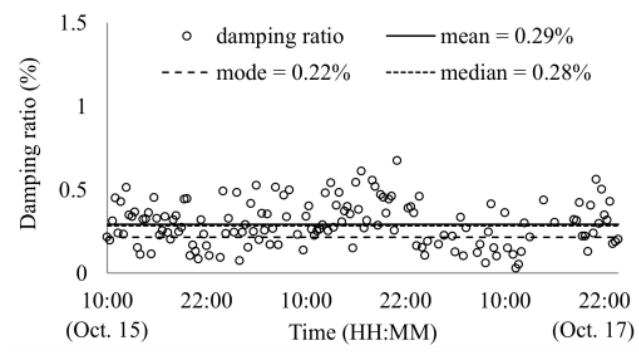

(b)

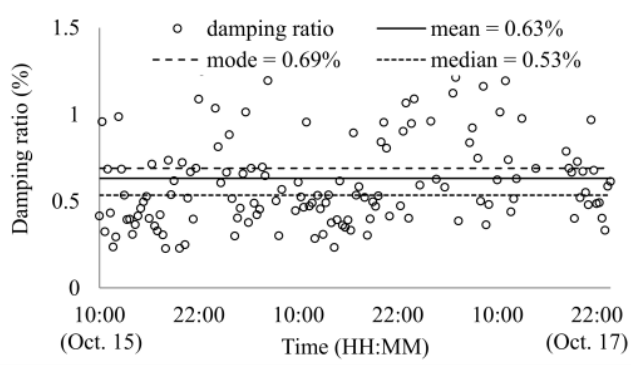

Fig. 9. Estimated damping ratio of (a) Bridge 2 and (b) Bridge 1 

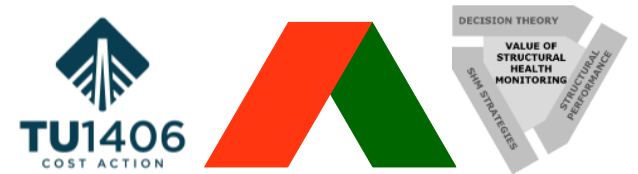

The Value of Structural Health Monitoring for the reliable Bridge Management

Zagreb 2-3 March 2017

\section{Case Study of Higher-Mode VIV in a Suspension Bridge}

\subsection{Observed VIV}

The Yi Sun-shin Bridge is a long-span suspension bridge with a main span length of 1,545m, adopting a streamlined twin-deck section. A VIV was observed in October, 2014 for a duration of two hours. At that time, one side of deck was closed for the replacement of epoxy-coated wearing surface. Being completely unaware of aerodynamic severance, the workers applied temporal covers on bridge railings for shielding the curing surface from chilly side winds, as shown in Fig. 10 (b). The time history of measured displacement is shown in Fig. 11. The maximum amplitude reached $0.52 \mathrm{~m}$ in double amplitude and the motional frequency was identified to be $0.3176 \mathrm{~Hz}$, which corresponded to the 4 th symmetric vertical mode of the bridge. The wind velocity was estimated to be $5.3 \sim 7.0 \mathrm{~m} / \mathrm{s}$ at the deck level.

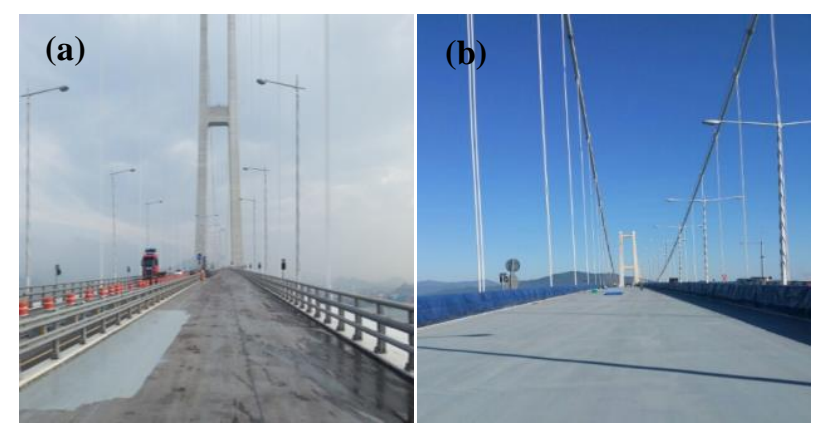

Fig. 10. Guardrails on decks (a): As-built, (b): Covered by temporal screens

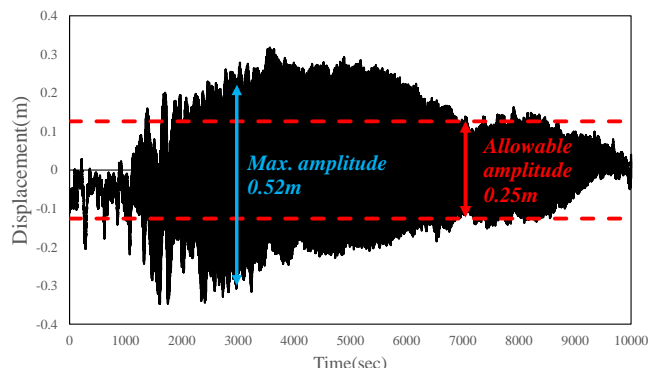

Fig. 11. Time history of VIV

\subsection{Examination of Temporal Screen Effect in Wind Tunnel}

A series of comparative wind tunnel tests were performed for two section models that simulating as-built and covered guardrails in Fig. 10. Since the amplitude of VIV was critically influenced by the level of modal damping ratio, the set-up damping ratios of the section models were variated for the evaluation of the dependency of VIV to damping ratios. Fig. 12 demonstrates a quite difference in aerodynamic performance for two guardrail conditions. The amplitude of VIV for the as-built guardrails is below the allowance even for the low damping ratio, which shows a stable performance in VIV. However, the guardrails covered by temporal screens leads a magnified VIV at the wind velocity of $4.4 \sim 8.3 \mathrm{~m} / \mathrm{s}$, which confirms to the measured wind velocity range of $5.3 \sim 7.0 \mathrm{~m} / \mathrm{s}$. Even in the design damping level, the amplitude remains in $0.55 \mathrm{~m}$ in a prototype scale, which correspondent to the measured one with $0.52 \mathrm{~m}$.

(a)

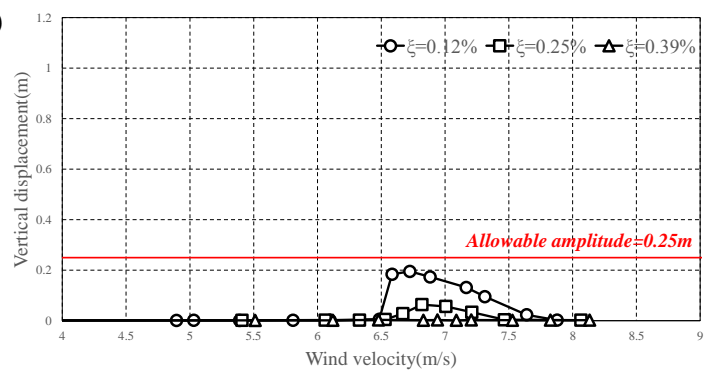

(b)

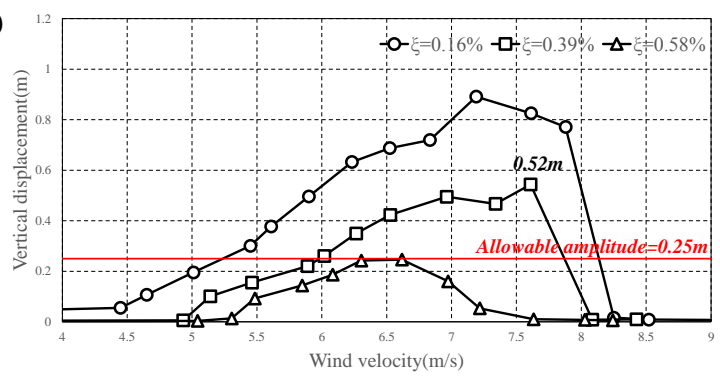

Fig. 12. Amplitude of VIV with (a) As-built guardrails, (b) covered guardrails temporarily

\subsection{Damping Estimation from Field-Monitoring Data}

To reveal a reason for higher mode VIV, modal damping ratios were identified by OMA. 1-hour ambient vibration data was utilized for damping identification. The Frequency Domain Decomposition (Brinker, 2000) and NExT-ERA were implemented for St-Id. The modal damping ratio of $4^{\text {th }}$ vertical mode was identified to be $0.42 \%$, which was equivalent to a design level, while the modal damping ratios for $1^{\text {st }}, 2^{\text {nd }}$ and $3^{\text {rd }}$ mode were evaluated as $1.26 \%, 2.42 \%$ and $0.44 \%$, respectively. This demonstrates that the happening event of VIV for the $4^{\text {th }}$ mode may be potentially related to a relatively low modal damping ratio for the $4^{\text {th }}$ mode. 


\section{Signal Stationarization in Damping Estimation}

Since the modal damping ratios were critical factors in assessment of vibrational serviceability in two demonstrated cases, the authors continued the investigation on the system identification approaches. The modal damping ratios can only be estimated when a bridge construction is being completed. Even though exciters have been utilized as a source of bridge motion for sweeping tests, it is not popular nowadays due to increased size of bridge structures. When the bridge is opened to traffic, the use of exciter is also not feasible when the traffic should be secured all the time. Accordingly, OMA is practically the sole approach we can choose, in which ambient vibration data are collected and modal damping ratios are identified by several St-Id techniques in operating condition. However, in contrast with the identification of natural frequency or mode shape, OMAbased damping estimation is still challenging due to several issues involved (Brewick and Smyth, 2014; Berwick and Smyth, 2014b; Chen et al., 2016; Guo et al, 2012; Kareem and Gurley, 1996; Seo et al., 2013; Kim et al., 2013; Kim et al, 2016; Rainieri and Fabbrocino, 2014; Tamura, 2012).

As was demonstrated in Fig. 9, the estimated damping ratios show a large scattering for each try, even though the most probable value may be represented as the average concept. One of the source of the scattering may be the nonstationarity in ambient vibration signals excited by moving vehicles. When the number of traffic lane over the bridge is only one or two and the traffic volume is not high, the ambient vibration signal at the sensor position show an envelope as a vehicle is approaching and fading away. Since OMA assumes that the signal to be analyzed is a stationary white-noise process, the envelop-like signal obtained from running vehicles can also contribute to scattering in St-Id. Fig. 13(a) shows a sample of traffic-induced vibration obtained from a bridge. The envelop-like responses show a certain level of nonstationary, as shown in Fig. 13(b).

(a)

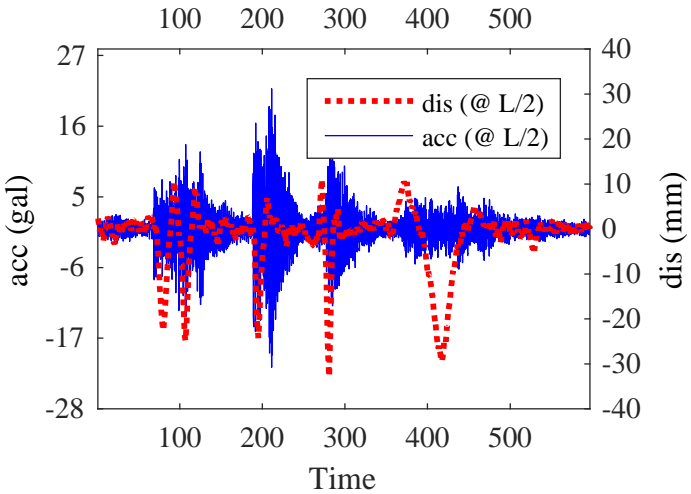

(b)

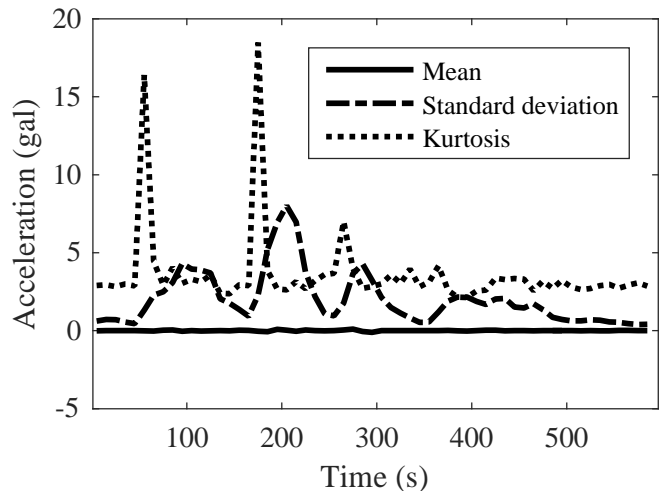

Fig. 13. (a) Measured displacement and acceleration at the center of mid span and (b) moving average, standard deviation and kurtosis of (a)

Lin and Chiang (2012) proposed a practical method to extract the approximate stationary process from nonstationary measurements containing envelope-like function. If the nonstationary loading can be represented by the product of amplitude-modulating function and stationary white noise process, then the envelope function can be evaluated by temporal root-mean-square function of responses. Consequently, the approximated stationary process can be extracted by dividing the measurements with calculated envelope function. Fig. 14 showed that this process reduced nonstationarity in traffic-induced response significantly

(a)

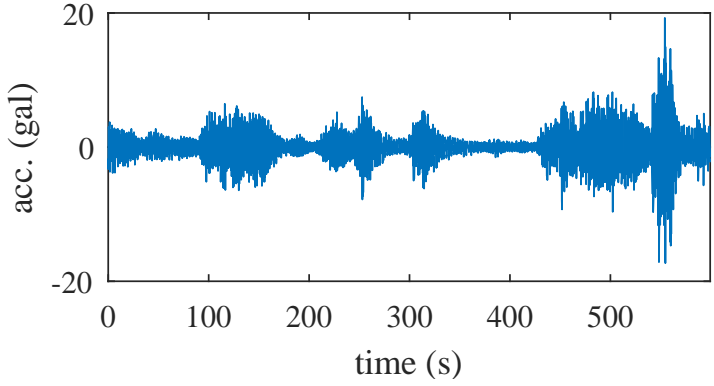

(b)

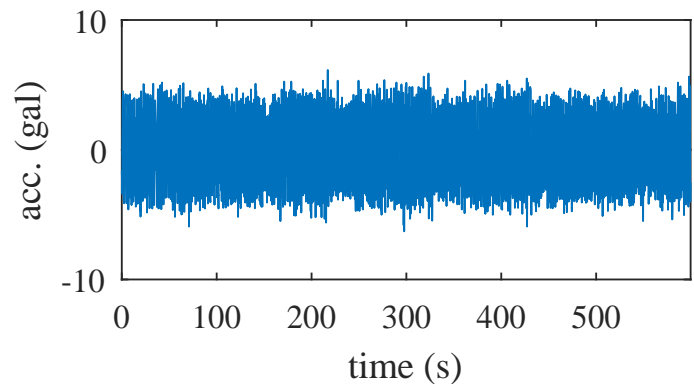

Fig. 14. (a) Measured acceleration and (b) approximated stationary acceleration 

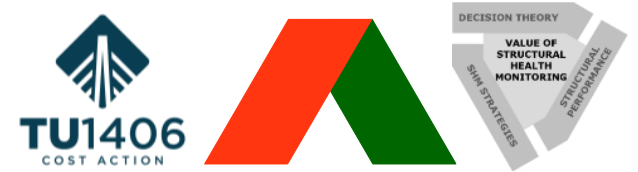

The Value of Structural Health Monitoring for the reliable Bridge Management

The signal stationarization process is applied to the same ambient vibration data utilized for the damping estimation in Fig. 9. The results are shown in Fig. 15. It is found that some highly scattered points were eliminated by the signal stationarization. The coefficient of variation (COV) of estimated damping ratios is reduced from $44.6 \%$ to $33.7 \%$, indicating the signal stationarization process is worth in reducing scattering in identified damping ratios from OMA.

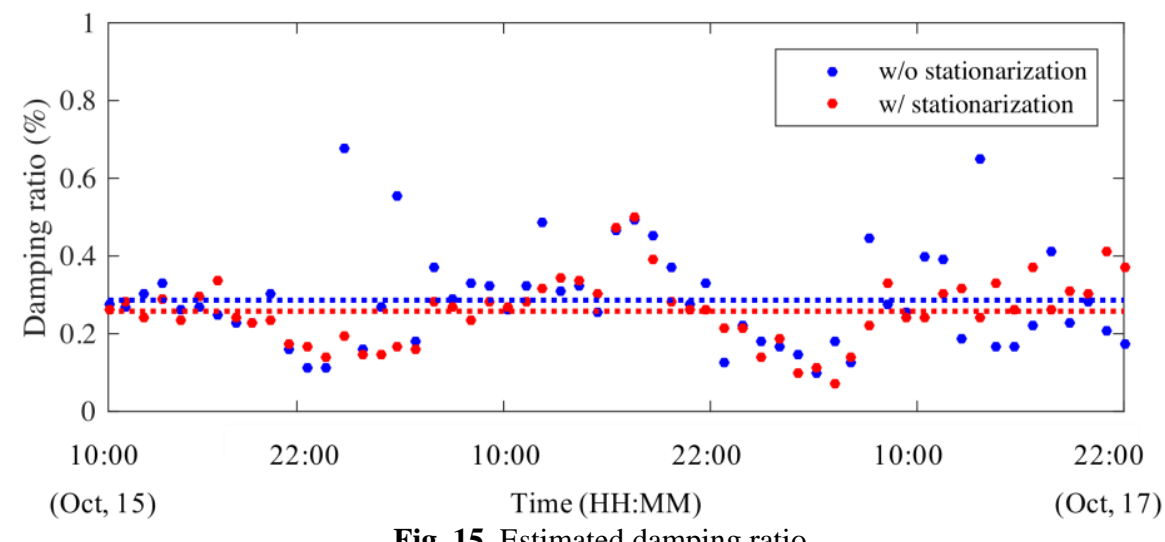

Fig. 15. Estimated damping ratio

\section{Concluding Remarks and Suggestions}

This paper investigated field-observed VIVs in two cable-supported bridges. One case study was for an interactive VIV in a twin cable-stayed bridge, the first observation in an actual bridge. The observable vibration in the upstream deck was induced by the magnified motion-induced vortex trails generated behind the deck due to the blockage effect by the downstream deck. Accordingly, the interactive VIV was identified to be sensitive to the open gap width between two decks. Meanwhile the close disposition between two decks was an indisputable cause of the observed interactive VIV, another issue of low damping ratio also attracted keen interest of engineers. The damping ratios for both bridges were identified from OMA for the field-monitored data. Even though both bridges were designed as a concept of twin bridges, the estimated damping ratios showed a wide difference. The damping ratio for the upstream bridge, subjected to interactive VIV, was estimated to be $0.28 \%$, while that of the downstream bridge was more than $0.6 \%$. Since the design damping ratio of the steel cablesupported bridge was set to $0.4 \%$ in Korean design guideline, the estimated damping ratio was much lower than expected. Another report of VIV in a super-long span suspension bridge was induced by an unexpected happening during the re-pavement work. However, it demonstrated that higher modes could be excited for this sort of very flexible structures and it might be correlated to the lower damping ratio among potential modes.

Two case studies demonstrate the importance of damping ratios in serviceability assessment and securing potential prognosis for enhancement of vibrational resilience for dominant modes. However, a large scattering in identified damping ratio remains to be a challenging issue to bridge engineers. The scattering can be induced from several factors such as nonstationary in monitoring data, but sophisticate and unpredictable relationship of damping ratio to many environmental factors such as amplitude of vibration, temperature change, source of vibration can make the identification of damping ratio to be more challenging by operational monitoring and modal analysis. This paper shows couple of efforts to approach this impending issues by introducing database for probabilistic assessment and data processing to eliminate large scattering in identified damping ratios. At the same time, the authors recommend to bridge owners to secure initial damping ratios for dominant modes and to confirm the validity of wind-resistance design in terms of design damping ratios. If necessary, the level of design damping ratio can be lowered to accommodate a potential possibility of low damping ratios of flexible structures, which adopt higher-strength materials and simpler connections.

\section{Acknowledgements}

This research was supported by the Grant (16SCIP-B119960-01) from the Ministry of Land, Infrastructure and Transport of Korean Government through the Korea Bridge Design and Engineering Research Center (KBRC) at Seoul National University; partial supports were received from the Korean Institute of Bridge and Structural Engineers (KIBSE) and the Hyundai Engineering and Construction. The authors particularly thankful to the Korea Infrastructure Safety \& Technology Corporation (KISTEK) and the JeollaNamdo for their sharing fieldmonitored data with us. 


\section{References}

Brewick, Patrick T., and Andrew W. Smyth. Exploration of the impacts of driving frequencies on damping estimates, Journal of Engineering Mechanics, 141(3), p.04014130, 2014.

Brewick, Patrick T., and Andrew W. Smyth. On the application of blind source separation for damping estimation of bridges under traffic loading. Journal of Sound and Vibration, 333(26), pp.7333-7351, 2014.

Brownjohn, James Mark William, Pilate Moyo, Piotr Omenzetter, and Yong Lu. Assessment of highway bridge upgrading by dynamic testing and finite-element model updating, Journal of Bridge Engineering, 8(3), pp.162-172, 2003.

Brownjohn, James Mark William, and Carden, E. Peter. November. Tracking the effects of changing environmental conditions on the modal parameters of Tamar Bridge, In 3rd international conference on structural health monitoring and intelligent infrastructure, 2007

Brincker, Rune, Ling mi Zhang, and P. Andersen., Modal identification from ambient responses using frequency domain decomposition, The 18th International Modal Analysis Conference, San Antonio, Texas, 2000.

Caicedo, Juan Martin, Shirley J. Dyke, and Erik A. Johnson., Natural excitation technique and eigensystem realization algorithm for phase I of the IASC-ASCE benchmark problem: Simulated data, Journal of Engineering Mechanics 130.1: 49-60, 2004.

Chen, Ge-Wei, Sherif Beskhyroun, and Piotr Omenzetter. Experimental investigation into amplitude-dependent modal properties of an eleven-span motorway bridge, Engineering Structures, 107, 80-100, 2016.

Guo, Y. L., A. Kareem, Y. Q. Ni, and W. Y. Liao. Performance evaluation of Canton Tower under winds based on full-scale data, Journal of Wind Engineering and Industrial Aerodynamics, 104,116-128, 2012.

Jin Park, Sunjoong Kim, and Ho-Kyung Kim. Effect of gap distance on vortex-induced vibration in two parallel cable-stayed bridges, Journal of Wind Engineering and Industrial Aerodynamics, Vol.162, pp.35-44., Available online at dx.doi.org/10.1016/j.jweia.2017.01.004, 2017.

Ju-Won Seo, Ho-Kyung Kim, Jin Park, Kwon-Taek Kim and Gi-Nam Kim. Interference effect on vortexinduced vibration in a parallel twin cable-stayed bridge, Journal of Wind Engineering and Industrial Aerodynamics, Vol. 116, pp. 7-20, Available online at dx.doi.org/10.1016/j.jweia.2013.01.014, 2013.

Kareem, A., and K. Gurley. Damping in structures: its evaluation and treatment of uncertainty. Journal of Wind Engineering and Industrial Aerodynamics, 59(2), pp.131-157, 1996.

Korean Society of Civil Engineers (KSCE). Design guidelines for steel cable-supported bridges, 2006.

Magalhães, Filipe, Álvaro Cunha, Elsa Caetano, and Rune Brincker. Damping estimation using free decays and ambient vibration tests, Mechanical Systems and Signal Processing, 24(5), pp.1274-1290, 2010.

Magalhães, Filipe, Álvaro Cunha, Elsa Caetano. Vibration based structural health monitoring of an arch bridge: from automated OMA to damage detection, Mechanical Systems and Signal Processing, 28, pp.212-228, 2012.

Peeters, Bart, and Guido De Roeck. One-year monitoring of the Z 24-Bridge: environmental effects versus damage events, Earthquake engineering \& structural dynamics, 30(2), pp.149-171, 2001.

Rainieri, Carlo, and Giovanni Fabbrocino. Operational modal analysis of civil engineering structures. Springer, New York, 142, p.143, 2014.

Sunjoong Kim, Ho-Kyung Kim, Radiance Calmer, Jin Park, Gyu Seon Kim and Deok Keun Lee. Operational field monitoring of interactive vortex-induced vibrations between two parallel cable-stayed bridges, Journal of Wind Engineering and Industrial Aerodynamics, Vol.123, Part A, pp.143-153, Available online at dx.doi.org/10.1016/j.jweia.2013.10.001, 2013.

Sunjoong Kim, Jin Park, Ho-Kyung Kim. Damping identification and serviceability assessment of a cable-stayed bridge based on operational monitoring data, Journal of Bridge Engineering, ASCE. 10.1061/(ASCE)BE.1943-5592.000100, Available online at dx.doi.org/10.1061/(ASCE)BE.19435592.0001004, 2016.

Tamura, Yukio. Amplitude dependency of damping in buildings and critical tip drift ratio. International Journal of High-Rise Buildings, 1(1), pp.1-13, 2012.

You-Chan Hwang, Sun-Joong Kim and Ho-Kyung Kim. Unexpected vibration monitoring in a suspension bridge and cause investigation, ACEM'16, ICC Jeju, Jeju island, Korea, August, 2016. 